GIL, J. A. Y J. MOLERO MESA -1984) Nueva cita de Riella helicophylla (Bory \& Mont.) para la Península Ibérica. Anal. Jard. Bot. Madrid 41 (1): 195-195.

GUERRA, J. E. RUIZ y C. SERGIO -1986- Sobre la distribución de Riella helicophylla (Bory et Mont.) Mont. en la Península Ibérica. Acta Bot. Malacitana 11: 75-76.

MOREIRA MADUEÑO, J. M (Edi.) -2004Caracterización ambiental de humedales en Andalucía. Consejería de Medio Ambiente. Sevilla ORTEGA, F., M. PARACUELLOS y F. GUERRERO -2004- Corología de macrófitos acuáticos en Andalucía oriental. Lazaroa 25: 179-185.

REYES PRÓSPER, E. -1910- Las carófitas de España. Singularmente las que crecen en sus estepas. Imprenta Artística Española. Madrid. $206 \mathrm{pp}$.

TALAVERA, S. P. GARCÍA MURILLO y H. SMIT -1986- Sobre el género Zannichellia L. (Zannichelliaceae). Lagascalia 14(2): 241-271.

Direcciones de los autores. ${ }^{1}$ Estudios de Flora y Vegetación (EFYVE) C/ Corregidor Nicolás $n^{\circ}$ 7, 29003 Málaga. ²Dpto. de Ecología y Geología, Univ.de Málaga, 29080 Málaga. ${ }^{3}$ Dpto. de Biología Vegetal, Univ. de Málaga, 29080 Málaga. ${ }^{4}$ EGMASA. P. C. Málaga Nostrum. Edif. Galia Center. C/ Jaén, 9-3 $3^{\text {a }}, 29004$ Málaga. ${ }^{5}$ Área de Ecología de la Univ. de Jaén. ${ }^{6}$ Consejería de Medio Ambiente, Junta de Andalucía. Oficinas de la Reserva Natural Laguna de Fuente de Piedra Cerro del Palo S/N 29520Fuente de Piedra (Málaga). *Autor para correspondencia: rconde@efyve.com.

\title{
183. NOVAS OCORRÊNCIAS DE SPHAGNUM AURICULATUM NO SUL DE PORTUGAL. SIGNIFICADO PALEOBIOGEOGRÁFICO
}

\author{
Carlos NETO $^{1 *}$, Pedro ARSÉNIO², Tiago MONTEIRO-HENRIQUES ${ }^{2}$, \\ Cecília SÉRGIO ${ }^{3}$ e José Carlos COSTA $^{1}$
}

Recibido el 21 de abril de 2009, aceptado para su publicación el 8 de junio de 2009 Publicado "on line" en junio de 2009

New chorological data of Sphagnum auriculatum in south Portugal. Paleobiogeographic meaning

Palavras-chave. Corologia, Sphagnum auriculatum, turfeiras de transição, sudoeste de Portugal.

Key words. Chorology, Sphagnum auriculatum, transition mires, southwestern Portugal.

O Sphagnum auriculatum Schimp. é uma espécie muito comum nas turfeiras baixas portuguesas e solos turfosos de linhas de água, especialmente no norte e centro de Portugal onde a precipitação média anual varia entre
$800 \mathrm{~mm}$ e $2800 \mathrm{~mm}$ (Séneca et al. 1992; Séneca, 1999; Séneca, 2003). No entanto, $S$. auriculatum perde em competição com outras espécies de musgos higrófilos (do mesmo género) nas turfeiras de altitude que tem muito 


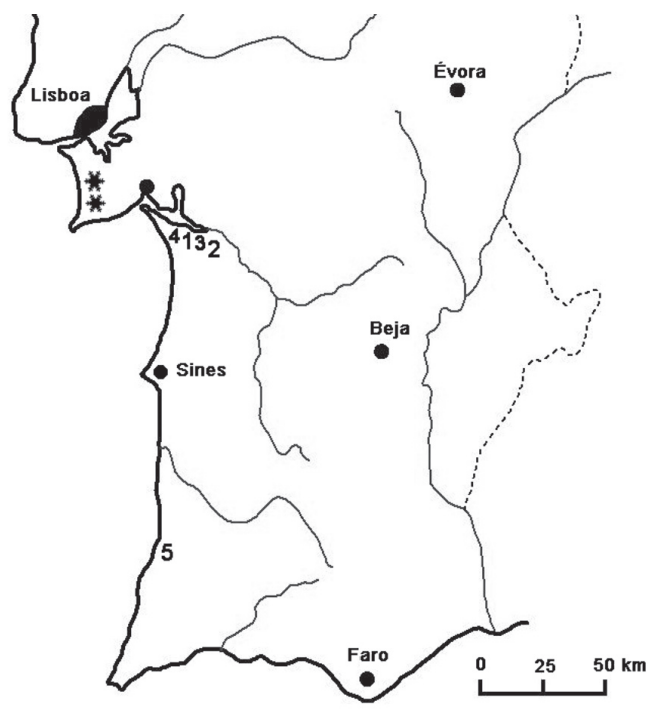

Figura 1 - Ocorrências de Sphagnum auriculatum no Sul de Portugal. Occurrences of Sphagnum auriculatum in the south of Portugal. $\star$ : Localizações anteriores a 1992. Os números correspondem a locais onde a planta foi encontrada em datas posteriores a 1992. 1. Lagoa da Batalha (1995) - $\left(38^{\circ} 23^{\prime} 24.82 " \mathrm{~N}\right.$; $8^{\circ} 34^{\prime} 53.71$ "W; alt: $\left.25 \mathrm{~m}\right)$; 2 . Texugueiras - Albergaria (1996) - (38 20'33.99"N; 8³3'27.65"W; alt: 27m); 3. Claros (Moinho da Ordem) (1997) - (38 20 20.32 "N; 8³3'03.36"W; alt: 24m); 4. Cachopos (1998) $\left(38^{\circ} 23^{\prime} 39.89^{\prime \prime} \mathrm{N} ; 8^{\circ} 39^{\prime} 44.56^{\prime \prime} \mathrm{W}\right.$; alt: 10m); 5. Arriba da Praia da Pedra da Bica (Zambujeira do Mar) (2009) - (37³1'53.68"N; 847'07. 51"W; alt: 42m).

menor representatividade em Portugal (Jansen, 2002; Honrado, 2001). Devido à sua particular resistência a temperaturas relativamente elevadas (dentro do género Sphagnum) tem sido encontrado em locais progressivamente mais setentrionais no território Português. Até 1992, a sul do rio Tejo, só eram conhecidas as ocorrências de Sphagnum auriculatum da Apostiça e da Lagoa da Casa (Sesimbra), figura 1 (Séneca et al. 1992; Queirós, 1985). Para Sul do Sado as localizações conhecidas até aquela data diziam respeito a restos fósseis (esporos e macrorestos) encontrados em grande quantidade nas acumulações de turfa que frequentemente colmatam algumas lagunas costeiras do Sado (Travessa, Carvalhal e Formosa), na região do Carvalhal-Comporta (Alcácer do Sal) (Mateus, 1985 e 1992). A partir de 1992 e até 1996 foi descoberta pela equipa da Faculdade de Ciências (José Mateus, Paula Queirós e Cecília Sérgio) uma turfeira no Sado com Sphagnum auriculatum vivo (Lagoa da Batalha - Alcácer do Sal; local 1 da figura 1) (Garcia et al. 2007). Posteriormente a espécie tem vindo a ser encontrada progressivamente mais para Sul, primeiro no Estuário do Sado: Texugueiras; Claros - Moinho da Ordem e Cachopos (Neto et al. 1996; Neto, 1997; Neto et al. 2005; Neto, 2002); e em 2009, pelos autores deste trabalho, na arriba da Praia da Pedra da Bica, na Zambujeira do Mar (Odemira, Baixo Alentejo). Esta última localidade fica a cerca de $98 \mathrm{~km}$ para sul da posição mais setentrional onde a espécie é conhecida em Portugal, correspondendo também ao local mais a sul de toda a sua área de distribuição na Europa (fig. 1). Neste último local o tapete de Sphagnum auriculatum com cerca de $1 \mathrm{~m}^{2}$ encontra-se numa plataforma rochosa a meio da arriba litoral com permanente circulação de água (fig. 2). A inacessibilidade do local justifica o facto de até agora não ter sido encontrado mas também porque deve ter uma ocorrência muito restrita.

No Sul de Portugal oSphagnum auriculatum está sempre associado directa ou indirectamente a acumulações arenosas antigas, de carácter dunar ou não, que ao induzir uma circulação hidrológica particular, permite a ocorrência de áreas permanentemente húmidas. Estes locais estão respectivamente associados a corredores interdunares com toalha freática superficial ou a arribas litorais xistosas que, ao cortarem os sistemas dunares que se estendem na superfície das plataformas litorais anexas, permitem uma lenta escorrência de água (fig. 2). Num caso e noutro a água da chuva infiltra-se directamente para a toalha freática subsuperficial (na base das dunas) sem escorrência superficial. Desta forma acumula-se água suficiente para alimentar permanentemente as escorrências ao longo das arribas e as depressões interdunares permitindo 

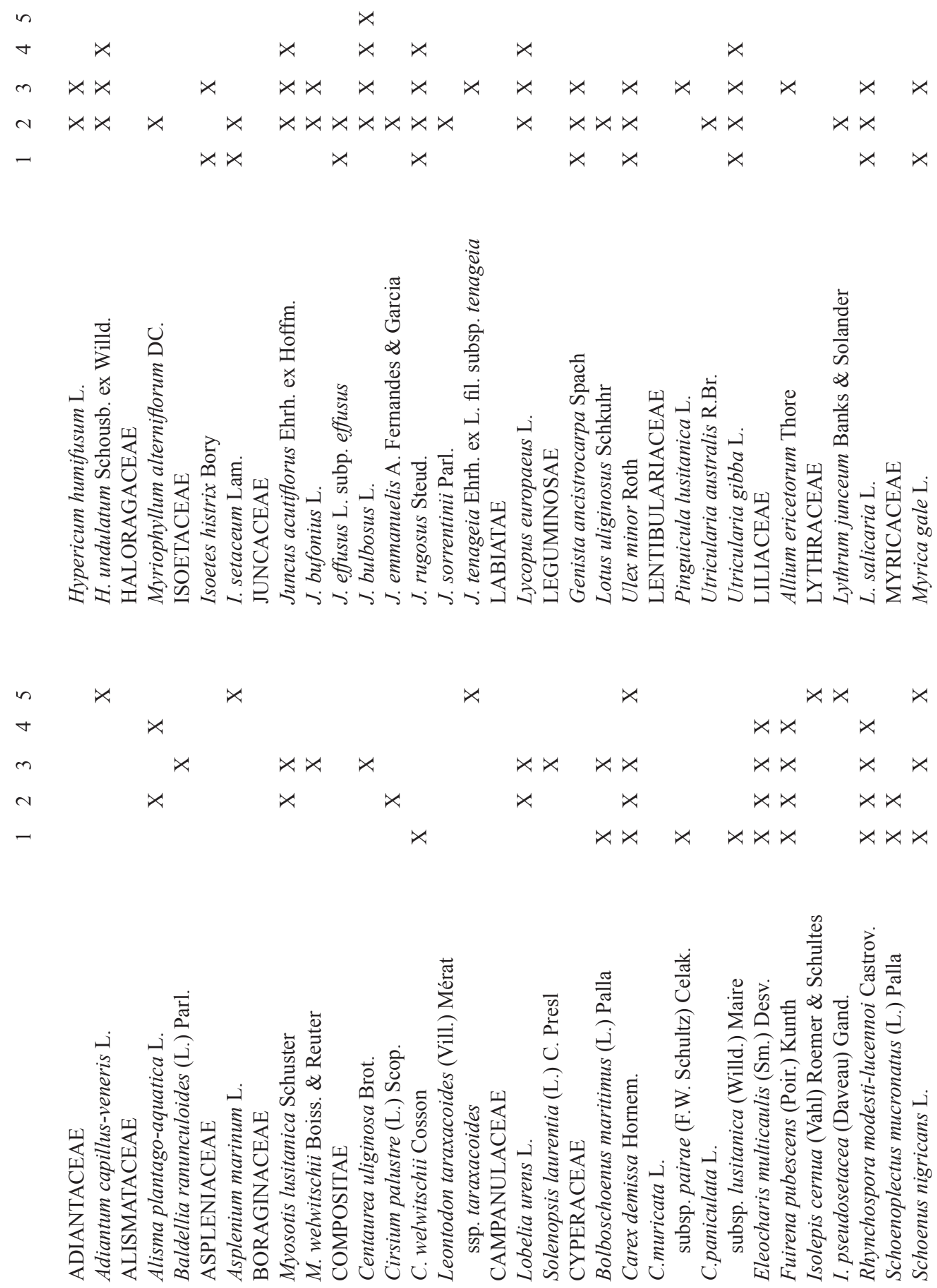


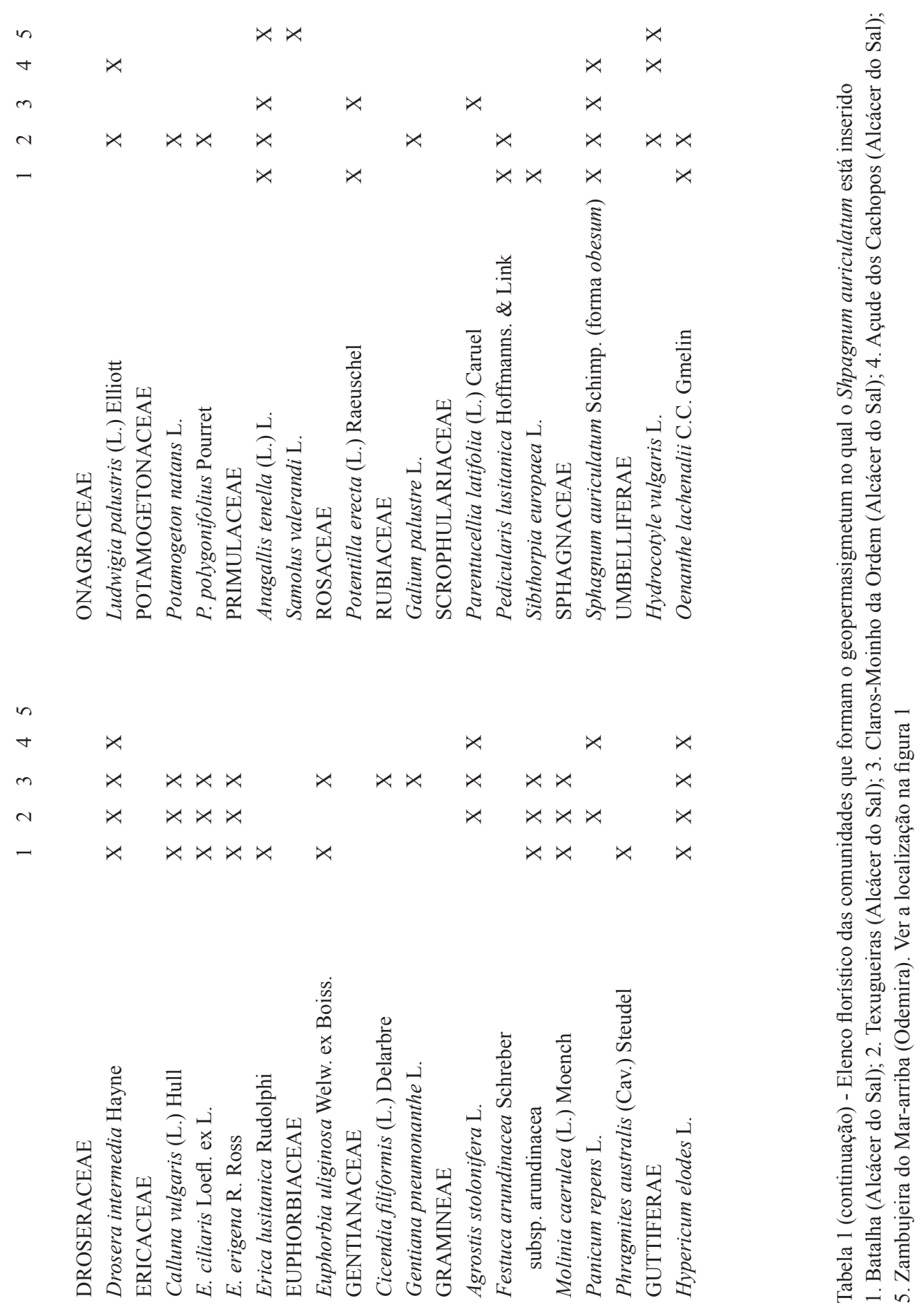




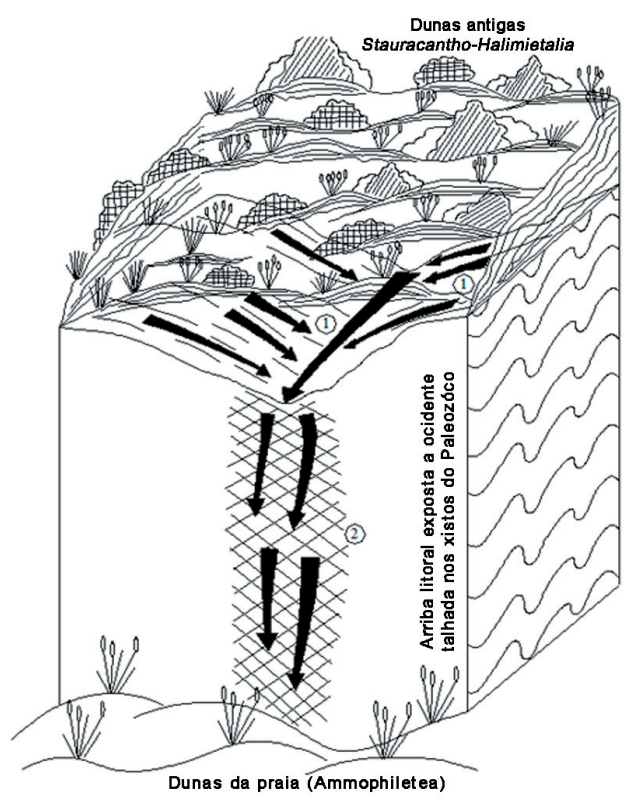

Figura 2 - Esquema de circulação da água nas arribas xistosas do sudoeste de Portugal. Scheme of water circulation in the Palaeozoic schist cliffs of southwestern Portugal. 1: Circulação hipodérmica da água (no contacto com a superfície dos xistos na base das areias antigas); 2: Arriba com escorrência lenta da água, durante todo o ano a partir da nascente que se forma no contacto entre a plataforma xistosa e as areias antigas. Nestas escorrências forma-se um geopermasigmetum onde convivem comunidades da Adiantetea; Isoeto-Littorelletea; IsoetoNanojuncetea, Potametea, Genistion micranthoanglicae, Scheuchzerio-Caricetea fuscae.

a ocorrência, nestes locais, de solos turfosos com "muck" e de comunidades vegetais turfosas. A originalidade deste esquema de circulação da água, num ambiente mediterrâneo, permitiu a subsistência de comunidades vegetais de carácter atlântico, que tiveram o seu óptimo durante os períodos frescos e húmidos do Quaternário e actualmente constituem uma vegetação relíquial (ilhas atlânticas em pleno mundo mediterrânico), (tab. 1). Deve referirse que o SW (Sado e Costa da Galé) tem em média menos de $700 \mathrm{~mm}$ de precipitação anual
(Daveau, 1977), valor que é inferior ao mínimo exigido por algumas das plantas que ocorrem nas turfeiras que se definem nos locais referidos anteriormente.

Os locais de ocorrência de Sphagnum auriculatum, apresentados na figura 1, são os únicos que se conhecem em todo o sul de Portugal. O geopermasigmetum turfoso que aí ocorre apresenta uma grande quantidade de comunidades vegetais de grande originalidade $\mathrm{e}$ caracteriza-se, floristicamente pela presença de espécies com óptimo Atlântico como (Cirsium palustre (L.) Scop., Drosera intermedia Hayne, Myrica gale L., Allium ericetorum Thore, Gentiana pneumonanthe L., Carex demissa Hornem., Rhynchospora alba (L.) Vahl, (local ined.), Euphorbia uliginosa Welw. ex Boiss.).

Por último deve referir-se a grande originalidade da ocorrência de Sphagnum auriculatum (forma obesum) acompanhada de Carex demissa, Anagallis tenella (L.) L., Juncus bulbosus L., Schoenus nigricans L., Isolepis cernua (Vahl) Roemer \& Schultes, Isolepis pseudosetacea (Daveau) Gand. nas arribas da Praia da Pedra da Bica. Aí a turfeira de Sphagnum convive em mosaico com comunidades briopteridofíticas da Adiantetea de grande originalidade onde, para além do Adiantum capillus-veneris L. e Asplenium marinum L. verifica-se a ocorrência dos briófitos Eucladium verticillatum (Brid.) Bruch \& Schimp., Eurhynchium speciosum (Brid.) Jur. e Didymodon spadiceus (Mitt.) Limpr. (Sérgio et al. 2006, Neto et al. 2007).

\section{BIBLIOGRAFIA}

DAVEAU, S. -1972- Répartition et rythme des précipitations au Portugal, Memória do C.E.G., Lisboa 3: 1-192.

GARCIA C., C. SÉRGIO, P. RODRÍGUEZGONZÁLEZ, C. RAMALHO, M. PORTO, M.J. PINTO \& S. LOBO -2007- Novas áreas para Portugal de Sphagnum auriculatum Schimp. e de Sphagnum subnitens Rusow \& Warnst. 
Portugaliae Acta Biolog 22: 208-209.

HONRADO, J., A. SENECA, F.B. CALDAS \& S.ORTIZ -2001- Complexos de vegetação turfófila nas serras do Parque Nacional da PenedaGerês (Subsector Geresiano-Queixense, Sector Galaico-português, Região Eurossiberiana). Quercetea 3: 197-211.

JANSEN, J. -2002- Guia Geobotanico da Serra da Estrela. Lisboa, ICN/PNSE.

MATEUS, J. E. -1985- The coastal lagoon region near Carvalhal during the Holocene; some geomorphological aspects derived from palaeoecological study at Lagoa Travessa, Actas da I. ${ }^{a}$ Reunião do Quaternário Ibérico 1 1: 237-251.

MATEUS, J. E. -1992- Holocene and present-day ecosystems of the Carvalhal region, southwest Portugal, Lisboa, tese de doutoramento.

NETO, C. -1997- A flora e a vegetação dos meios palustres do Superdistrito Sadense. Lisboa, Centro de Estudos Geográficos.

NETO, C. -2002- A Flora e a Vegetação do superdistrito Sadense (Portugal). Guineana 8: 1-269.

NETO, C., J. CAPELO, J.C. COSTA \& M. LOUSÃ -1996- Sintaxonomia das Comunidades de turfeira do Superdistrito Sadense. Silva Lusit. 4: 257-258.

NETO, C., M. E. MOREIRA \& R. M. CARAÇA -2005- Landscape Ecology of the Sado River Estuary (Portugal). Quercetea 7: 43-64.

NETO, C., J. CAPELO, C. SÉRGIO \& J. C. COSTA -2007- The Adiantetea class on the cliffs of SW Portugal and of the Azores. Phytocoenologia 37(2): 221-237.

PEREIRA, M. D. \& C. NETO -2008- Primeiros Subsídios ao Elenco Florístico e às Comunidades Anfíbias no Sul de Portugal - Bacia Hidrográfica do Rio Sado. Acta Bot. Bras. 22(3): 771-781.

QUEIRÓZ, P. F. -1985- Dados para a história holocénica da região da Lagoa de Albufeira sumário das conclusões do estudo paleoecológico da Estacada. Actas da I. ${ }^{a}$ Reunião do Quaternário Ibérico 1: 251-263.

SÉNECA, A. -1999- Estudo Ecológico e Biossistemático do Género Sphagnum L. em Portugal. Dissertação de doutoramento. Faculdade de Ciências da Universidade do Porto.
SÉNECA, A. -2003- The genus Sphagnum L. in Portugal. Cryptogamie, Bryologie, Lichenologie 24(2):103-126.

SÉNECA, A., C. SÉRGIO, P. QUEIRÓZ \& J. MATEUS -1992- Sphagnum auriculatum Schimp. in Portugal with late Quaternary occurrences. Osiris 7:11-20.

SÉRGIO C., C. GARCIA \& C. NETO -2006- New interesting mosses occurring on moist calcareous cliffs in West Coast of Portugal. Silva Lusit. 14(2): 265-279.

Dirección de los autores. 'Instituto de Geografia e Ordenamento do Território .University of Lisbon. Alameda da Universidade. 1600-214 Lisboa. PORTUGAL. ${ }^{2}$ Instituto Superior de Agronomia. Technical University of Lisbon (TU Lisbon). Tapada da Ajuda. 1349-017 Lisboa. PORTUGAL. ${ }^{3}$ Museu Nacional de História Natural, Jardim Botânico/CBA. University of Lisbon. Rua da Escola Politécnica, 58. 1250-102 Lisboa. PORTUGAL

*Autor para correspondencia: carlosneto@fl.ul.pt. 